\title{
Training Licensed Nuclear Power Plant Operators for Handling Unforeseen Accident Events: Development and Assessment of a Training Approach Based on Adaptive Expertise Theory
}

\author{
Ann Britt Skjerve* and Lars Holmgren
}

Institute for Energy Technology, Halden, Norway

\begin{abstract}
Licensed nuclear power plant (NPP) operators should be able mitigate any safety-critical situation that may lead to major accidents in NPPs. Training aimed at mitigating standard accident events is well-established and fully supported by operating procedures. This study focused on training aimed at unforeseen, i.e. non-standard, accident events. In these situations, procedure compliance may not be possible and/or may not take the plant to a safe state, and for this reason the classic approach for training NPP operators, which emphasised over-learning and procedure compliance, is not applicable. The purpose of the study was to develop and investigate the potential applicability and usefulness of the training approach "Coaching for Improved Ability to Handle Unforeseen Events" (CIAU). CIAU was developed based on adaptive expertise theory, using constructivist training techniques, such as exploration and critical thinking. CIAU was assessed using a full-scale, graphic control-room simulator. In all, 21 licensed NPP operators participated in the study. Each operator performed one scenario. The operators had no operating procedures available, except for a few procedure steps. Data collection covered video and audio data and assessment made by coaches and trainees. The study showed that both trainees and coaches judged that CIAU training succeeded in promoted the quality of the strategies developed by the trainees to manage unforeseen events. It suggested that CIAU training deepened the trainees conceptual understanding of plant processes, and thus facilitated transfer of knowledge across the various operational events. The aspect of the CIAU training the trainees appreciated most was that they were allowed to develop a strategy on their own, and thus came to understand all aspects of the strategy in-depth. Overall, the outcome of the study suggested that CIAU training is an applicable and useful training approach that may potentially supplement the classic approach for training NPP operators.
\end{abstract}

Keywords: Training; Unforeseen events; Adaptive expertise; Nuclear power plants

\section{Introduction}

Modern societies comprise organisations with potentials for major accidents. These organisations are built to generate products or services of crucial importance to the society, such as delivering energy and transporting people and goods. Major accidents may be described as events in which the normal workings of an organisation break down and novel unforeseen accident situations arise. Various theories have been proposed to account for how to protect against major accidents. These theories include, the Energy and barrier perspective [1,2], Manmade disasters [3-5], Normal Accident theory [6], High-Reliability organisations [7], Conflicting-objectives perspective [8], and Resilience engineering [9].

Nuclear power plants (NPPs) contribute with about $11 \%$ of the world's electricity using around 450 power reactors [10]. They are among the organisations with potentials for major accidents, as sadly testified by history [11]. To protect against major accidents, NPPs are constructed and organised based on the Defence-In-Depth strategy [12]. This implies that independent and redundant defences are used to protect against radioactive releases to the environment.

The operational activity in NPPs is highly proceduralised. Today, operating procedures exist for all standard tasks, such as plant startup, normal operation and plant shut-down, as well as for abnormal situations and emergencies, and thus accident events that have been foreseen to occur with a certain degree of probability. The emergency operating procedures were largely developed following the Three Mile Island accident [13] in 1979, and later refined based on the outcome of plant-specific Probabilistic Safety Assessments [14]. The high level of proceduralisation implies that licensed NPP operators both in their daily work and during refresher training courses, i.e. training courses performed regularly throughout their career focus strongly on procedure implementation.

To ensure predictability and maintain control of NPPs, it is important that operators comply with the operating procedures. Still, if the emphasis on procedure compliance becomes too strong, operators may be at risk for developing a checklist mentality. A checklist mentality implies that operators will focus more on how to perform a task, rather than why they perform a task, that they will focus on the procedures rather than building on integrated understanding of the plant and its processes. This reality of this risk might be reflected in studies of event analysis performed by the IAEA, which showed that insufficiency in "understanding of nuclear/process safety issues in decision making and actions" were among the most consistently found root causes [15].

*Corresponding author: Ann Britt Skjerve, Institute for Energy Technology, Halden, Norway, Tel: +47-69212230; E-mail: ann.britt.skjerve@ife.no

Received July 04, 2018; Accepted July 16, 2018; Published July 21, 2018

Citation: Skjerve AB, Holmgren L (2018) Training Licensed Nuclear Power Plant Operators for Handling Unforeseen Accident Events: Development and Assessment of a Training Approach Based on Adaptive Expertise Theory. Arts Social Sci J 9: 385. doi: 10.4172/2151-6200.1000385

Copyright: (C) 2018 Ann Britt Skjerve, et al. This is an open-access article distributed under the terms of the Creative Commons Attribution License, which permits unrestricted use, distribution, and reproduction in any medium, provided the original author and source are credited. 
Operating procedures are developed based on a set of preconditions. If the pre-conditions fail, procedure compliance may not be possible and/or may not take the plant back to a safer state. In these situations, it is important that operators are able to assess the situation at hand without the assistance provided by the procedures and to develop a strategy for how to proceed. Still, it is rare that operators need to "take over" from the procedures in this way, as pre-conditions usually are fulfilled. This creates a condition that mimics the paradox Bainbridge [16] identified for highly-automated systems: The more extensive and robust the operating procedures are, the less practice operators will get in handling tasks without the guidance provided by the procedures, and the more training they need to uphold this competence.

Unforeseen events are non-standard and unique. They have characteristics that cannot easily be predicted based on historical data and experience [17]. They may develop into major accident events in a short time-span, as was witnessed in the Fukushima accident [18]. For this reason, a key question is how to train operators to be prepared for handling unforeseen events. Adaptive expertise theory [19] offers a potential basis for developing this type of training. Adaptive expertise is expertise in solving non-standard tasks and has traditionally been seen as encompassing three elements: domain-specific skills, metacognitive skills and innovative skills. Adaptive expertise promotes the trainee's ability to engage in conceptual reasoning and implies that existing knowledge is deconstructed and reconstructed in new ways, allowing it to be transferred to and applied in novel, non-standard events. Adaptive expertise implies slow thinking [20], as it is conscious and calculated in nature, and reflects knowledge-based reasoning processes [21].

The study presented in this paper was performed to investigate the applicability and usefulness of a training approach based on application of adaptive expertise theory [19] and using constructivist learning principles [22] for promoting trainees' ability to handle unforeseen accident events in NPPs.

The paper documents the development of the training approach called "Coaching for Improved Ability to Handle Unforeseen Events" (CIAU) and an assessment of its potential usefulness for promoting licensed NPP operators' ability to handle unforeseen events. CIAU was designed by the Halden Reactor Project [23], as a continuation of an earlier study [24].

CIAU training differs markedly from the approach that has traditionally been used to train licensed NPP operators: The traditional approach implies that an operating crew is jointly trained in a replica full-scale plant-specific simulator, where they face accident scenarios designed to allow practicing of one or more particular emergency operating procedures. A key advantage of this training approach is that crew members practice handling of accident situations jointly in as realistic a setting as possible. A disadvantage is that the trainees do not necessarily fully understand how the scenario was managed and/ or why certain decisions were made. One reason for this is that the individual trainee will have to attend to many tasks that may interfere with his or her possibility for engaging in reflections during the scenario: Following the operating procedure is in itself a demanding task, contributing to crew communication and the crew's decisionmaking processes, logging activities, etc. Also, the debriefing session may not cover or not cover in sufficient detail for the individual trainee all topics the trainee has not fully understood.

CIAU training, on the other hand, implies training of an individual operator in a graphic full-scale plant-specific simulator, where the trainee faces accident scenarios designed to make it necessary for the trainee to adapt the operating procedures to the situation at hand. The trainees are instructed to develop a solution strategy - using exploration and experimentation and critical thinking - that will take the plant back into a safer state. In CIAU training all factors that may prevent or disturb the trainee's reflections on the events contained in the scenario are removed: there are no crew members, no procedures except for particular procedure steps, and no requirements for logging or other administrative tasks. The reflections of the trainee are further reinforced by the addition of two coaches that will guide the trainee's reasoning processes, if needed. The disadvantage of CIAU training is thus that the setting is less realistic than traditional training. The advantage is that the trainees will develop a detailed conceptual understanding of the scenario elements addressed in a training session.

The evaluation of the potential usefulness of CIAU for promoting licensed NPP operators' ability to handle unforeseen events was carried out using a graphic full-scale plant-specific training simulator and 21 licensed NPP operators took part in the evaluation process. It addressed three research questions:

- Research Question 1: Does CIAU training show a potential for promoting licensed NPP operators' ability to handle unforeseen events?

If research question 1 was answered confirmatory:

- Research Question 2: What competencies did the trainees improve?

- Research Question 3: What aspects of training did the trainees appreciate most and what aspects did the find most demanding?

The study demonstrated that CIAU training has potentials for promoting licensed NPP operators' ability to handle unforeseen by advancing adaptive expertise, and thus that adaptive expertise theory successfully can be applied in the domain of NPP operation. It further demonstrated that the CIAU training approach is highly applicable. Based on the outcome of the study, it is argued that training aimed at promoting adaptive expertise may be a useful supplement to the classic approach for training of NPP operators. Even though the CIAU training approach was developed for the domain of NPP operation, it is generic in nature and may likely be applicable in all domains where operation is highly proceduralised. Publishing the findings from the development and evaluation of CIAU training, is an important step in making the training method known for different organizations.

\section{Adaptive Expertise}

Adaptive expertise is a broad psychological construct referring to professional expertise that enables a person to handle non-standard tasks within a given domain. Adaptive expertise theory $[19,25]$ holds that for a person to become an adaptive expert, he or she must thus first develop routine expertise. Routine expertise is manifest when a person can perform standard tasks using well-learned solution strategies with high efficiency and accuracy within a given domain. Routine expertise is developed based on extensive experience with implementing prescribed solution strategies, often in the form of procedures. Still, even though people with routine expertise perform standard tasks well, they do not necessarily understand why the pre-scribed solution strategies work. For this reason, it is challenging for routine experts to adapt their performance strategy when unforeseen events arise, and the standard solutions do no longer apply. Routine expertise is developed using training principles such as overlearning, offering step-by-step guidance 
and providing feedback that emphasises compliance and accuracy [26]. These training techniques are all characterised by limiting learner control over the training session.

Adaptive expertise is expertise in solving non-standard tasks and thus tasks that has not been foreseen to occur. Traditionally, this type of expertise has been seen as comprising three distinct elements [19,25]: Domain-specific skills, metacognitive skills and innovative skills. A person with adaptive expertise has conceptual knowledge of the domain, which in adaptive expertise theory has been defined as knowledge of the "how and why" each step of a procedure works [19]. Conceptual knowledge is represented in high-level, abstract, cognitive structures [27], which enable adaptive experts to transfer and adapt their knowledge to novel situations [28]. Adaptive experts are able to identify situations in which then standard solutions do not apply and to develop novel solution strategies [29]. Bell and Kozlowski [26] argue that adaptive experts are able to view situations from the outside, to draw analogies to other situations, and to develop innovative solutions by decomposing their existing knowledge into its basis elements and flexibly to flexibly recompose the knowledge elements in new ways to solve novel problems [30].

The training techniques associated with development of routine expertise to solve standard tasks will not be suitable for developing adaptive expertise [27]: With continued training for routine expertise, a trainee may be able to perform the standard tasks still slightly faster and with still slightly greater accuracy and automaticity, but it will not lead to expertise in solving non-standard tasks. For adaptive expertise to develop, a person needs to engage in conceptual thinking. He or she needs to focus on why and under what conditions the standard solutionstrategies (e.g. operating procedures) do and do not apply. To promote the development of adaptive expertise, the use of principles based on constructivist learning theory [22], and thus active, learner-centred approaches, are generally recommended $[26,29]$. Training developed to promote adaptive expertise should be designed to encourage trainees to engage in exploration, experimentation and engage in critical thinking, i.e. "... analyzing and evaluating thinking with a view to improving it" $[30,31]$, to develop their own solution strategies to novel, unforeseen events. It has been speculated that constructivist learning principles will be effective to achieve this purpose, because they allow trainees directly, without any mediation, to integrate the knowledge they gain into their existing knowledge structures [27]. Advance organisers are abstract ideas that draw on anchoring concepts, which are already in the trainee's mind [32]. They may take the form of e.g., comparative organizers, such as schemes to help compare the characteristics of different situations or objects or analogies. Advance organisers may further be used to support trainees in linking novel knowledge to their existing knowledge and thus to promote the development of adaptive expertise.

\section{Coaching for Improved Ability to Handle Unforeseen Events (CIAU)}

The training approach "Coaching for Improved Ability to Handle Unforeseen Events" (CIAU) was developed to advance adaptive expertise in the population of licensed NPP operators in order to promote their ability to handle unforeseen events. CIAU targets problem-solving skills, not perceptual-motor skills. CIAU training is designed to be carried out in a graphic full-scale plant-specific training simulator. The trainees participate individually and receive coaching from two coaches. The trainees have no operating procedures available, except a few procedures steps. They are encouraged to develop solutions strategies for managing novel, unforeseen accident events by engaging in exploration, experimentation and critical thinking.

\section{Basis for CIAU training}

The CIAU training approach is designed with the intent to cover the three elements traditionally associated with adaptive expertise (above Section): It focuses directly on promoting the trainees' conceptual understanding of the specific domain and on the trainees' metacognitive skills, i.e. skills associated with awareness and control of own cognition [33]. Jointly improvements associated with these elements, are intended to advance the trainees' ability to develop novel solution strategies and thus their innovative skills.

CIAU training makes use of constructivist learning theory as a basis for promoting adaptive expertise [22]. It is an active learning approach, in the sense that it "... involves students in doing things and thinking about the things they are doing" and falls within the category of discovery learning $[34,35]$. When learning something new, people will strive to relate their new knowledge to their existing knowledge, which implies that they organise and integrate the training material into their current knowledge structure [36]. CIAU aims at supporting trainees in organising their knowledge in "meaningful structures" [29] by the use of coaching, and thus constitutes a guided discovery learning approach. The coaches will, e.g., assist trainees in sequencing the problems address, as opposed to jumping to and from partly solved problems or facing all problems at once. This is done to reduce the risk that factors such as mental-overload and learning confusion will come to hinder or prolong learning [37].

Vygotsky's theory on the zone of proximal development which was originally developed to support education of children, served as basis for developing guidance to the coaches on how to organise the coaching process [38]. Vygotsky defined the zone of proximal development as "...the distance between the actual developmental level as determined by independent problem solving and the level of potential development as determined through problem-solving under adult guidance or in collaboration with more capable peers" [38]. In the CIAU training, what may be characterised as a trainee's zone of proximal development is determined for each scenario based on the trainee's entry competence vis-à-vis the topical areas covered in a particular scenario (see further below). This approach implies that the trainees engaged in a CIAU training sessions are not expected to master the same training material. The only common learning goal for all trainees is that they should improve their level of adaptive expertise.

Trainees participate individually in CIAU training and are asked to think aloud and to respond to the guiding questions of the coaches, which will gradually be more challenging. This environment has the potential to be very stressful to the trainees. Studies have shown that adult learning processes may be inhibited if the learner becomes overanxious, and an important element that contributes to anxiety is fear of what others might think of them [39]. For this reason, it is important that the coaches establish a learner-friendly training environment. This implies that it is continuously clear to the trainee that he or she is engaged in learning, in generating and re-structuring knowledgeas opposed to being in a test situation where the goal is to perform "correct".

A CIAU training is intended to comprise collection of data to allow assessment training of effectiveness. The lessons learned from training sessions may warrant adjustments in the training approach, including in the design of the training scenarios applied. 
Citation: Skjerve AB, Holmgren L (2018) Training Licensed Nuclear Power Plant Operators for Handling Unforeseen Accident Events: Development and Assessment of a Training Approach Based on Adaptive Expertise Theory. Arts Social Sci J 9: 385. doi: 10.4172/2151-6200.1000385

\section{Preparations for CIAU training}

The activities involved in preparing for CIAU training is shown in Figure 1. They are organised under two headings. Generic preparations are essentially the same from one training session to the next, and unless changes are introduced, e.g. new instructors. Scenario-specific preparations refer to preparation that need to be performed for each scenario to be used in a CIAU training session.

Generic preparations: Training of coaches: The coach must be adaptive expert with respect to the plant addressed in the training session, and understand the system numbers, abbreviations, etc. used by the trainees. This is necessary, as the coaches should be able to follow and flexibly adapt guidance to the reasoning processes of the trainee. The coaches should reckon the importance of encouraging the trainees to take responsibility for the learning process. They should master a dialogue technique implying that they begin guidance processes with open general questions and only gradually use more and more pointed questions to help the trainee achieve the specific insights, if needed. This technique also implies assisting the trainees in systematically assessing advantages and disadvantages of affordable options prior to making a decision. The coaching process is in itself intended to serve as an example to the trainees on how they may approach unforeseen nonstandard situations. Having an approach available for how to approach an unforeseen event may reduce the risk that trainees' performance will be impacted by negative stress-reactions, such as tunnel-vision, reduced search for information, and impulsive actions [40], should an unforeseen event arise in the normal work environment.

Preparing evaluation forms: Two different evaluation forms are used as an integrated part of a CIAU training session: One form is filled-in by the trainees and the other by coaches individually. The purpose of the evaluation forms is to obtain data to support assessment of training effectiveness and potential for improvements. The questionnaires contain 9-10 generic items that are suitable for all scenarios. The trainees respond to a subset of the items during a scenario break (see below), and the reminder following scenario completion. The items cover the individual trainee's satisfaction with/ ability to learn from CIAU training, the content of the training material learned, the trainee's perception of scenario novelty, and the impact of training as assessed subjectively, and the coaches' assessment of the quality of the trainee's strategy. Referring to Kirkpatrick's framework for assessment of training effectiveness [41], which is probably the most widely used assessment framework today $[42,43]$, the data should support assessment of level one (reactions) and two (learning).

Preparing debriefing: After the training session, the trainee should be invited to take part in a debriefing session. This may be an individual debriefing session or a joint session with a group of trainees that all have faced the same scenario(s). Debriefing should be carried out using the guidance technique applied to coach the trainees. The overall purpose of the debriefing session is to reinforce the lessons learned by the trainees and to promote positive training transfer. Preferably training transfer should imply that they use same approach as applied by the coaches to question and reflect upon everyday occurrences in the plant and thereby continuously deepen and expand their conceptual understanding of the plant processes.

Specific preparations: Training needs: A training needs analysis may be carried out using the technique usually applied at the plant, where training is carried out, e.g., based on recommendations in the Systematic Approach to Training as has been recommended by the IAEA [44]. CIAU training is suitable for addressing training needs concerned with promoting trainee's ability to handle unforeseen events. A CIAU training scenario may be designed to target selected "operator fundamentals" [45], such as break dynamics, heat transfer, thermal hydraulics, and reactivity control, depending on the training needs identified.

Scenario design: CIAU scenarios should comprise events that are novel to the trainees and cannot be handled without adapting the operating procedures. To develop emergency scenarios, which licensed NPP operators will perceive as completely novel, is highly challenging. All licensed NPP operators regularly engage in training of "standard" emergency situations, i.e. scenarios which can be handled with limited or no adaptations of the operating procedures. One way to develop scenarios that will be perceived as novel by licensed NPP operators, and imply that the operating procedures are adapted, is to apply unforeseen combinations of two or more "standard" scenarios, and if necessary by adding further malfunctions and/or deviations to the scenario. This design approach implies that some scenario elements will be familiar to the trainee, even though the situation as overall should be perceived as novel. An additional requirement to CIAU scenarios is that they should be sufficiently complex and comprehensive to allow all trainees from the specified population to expand their level of adaptive expertise, regardless of their entry level.

Operator profiling and topical area guidance: A CIAU training session implies that the coaches jointly develop an operator profile for each trainee for each scenario applied. A profile is thus scenario specific. It is developed based on the characteristics of the strategy developed by the trainee for how to handle an event prior to receiving coaching (Figure 2), including the trainee's answers to five generic questions, which are intended to capture aspects of importance for understanding the trainees reasoning processes, which cannot be expected to be expressed during the think aloud session. These five questions are: (1) How serious do you think the situation is? (2) How confident are you that the strategy you developed will take the plant into a safe state? (3) What plant parameters were the most important when you developed your strategy? (4) Have you experienced similar situations (as the one faced in the present scenario) in simulator training sessions before? And (5) Would any other strategies than the one you developed have succeeded in bringing the plant to a safe state? The operator profile

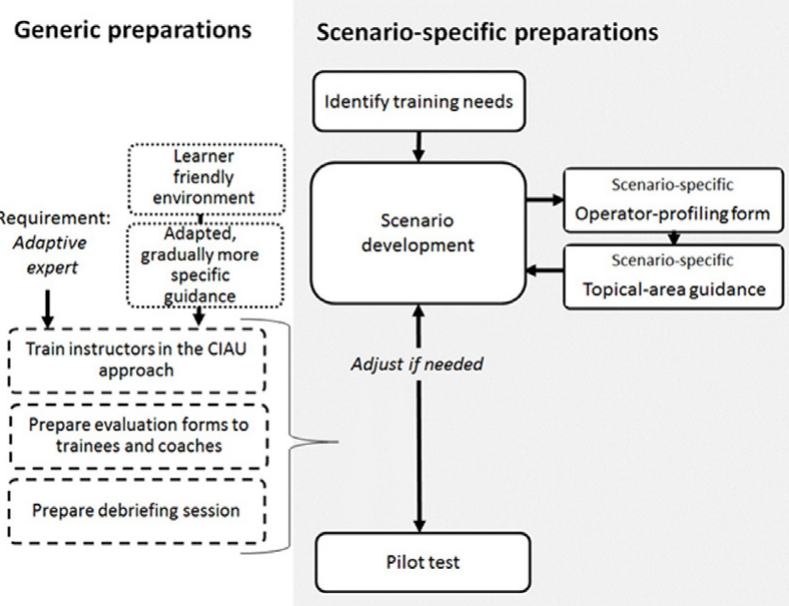

Figure 1: Overview over the preparations required to carry out CIAU training: The left-hand side of the figure shows generic preparations that are similar across training sessions. The right-hand side of the figure shows scenariospecific preparations. 


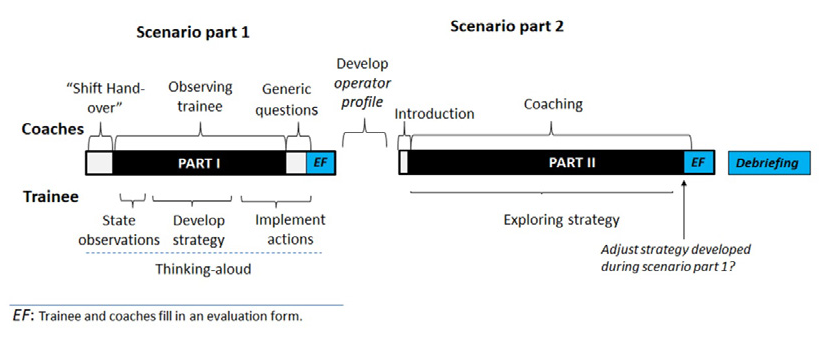

Figure 2: Overview of the content of a CIAU training session. "EF" refers to periods where the trainees and coaches fill-in evaluation forms.

is developed using a scenario-specific form, which documents the scenario elements (e.g. parameters such as the level and flow in a tank) and the recovery-strategy characteristics the trainee should preferably notice/employ to handle the event. Based on the operator profile, the coaches then use the Topical Areas form to decide what topics that should be addressed in the coaching process and in what depths - a decision that may later be revised still guided by the Topical Area form. This process implies that the material the trainees are expected to master may vary from one trainee to the next, depending on their entry level of understanding. The only common learning goal for all trainees is that their level of adaptive expertise should be improved.

Pilot testing and adjustments: Prior to initiating training, the scenarios, as well as the associated training material, should be exposed to a pilot test. This test will ensure that the scenarios function as intended - possibly following some adjustments and provide the coaches with more insights into what topics and issues trainees may likely address, and thus help the coaches to prepare for the training session.

\section{Test Procedure}

\section{Training facility}

The evaluation of the potential usefulness of CIAU for promoting licensed NPP operators' ability to handle unforeseen events was carried out using a full-scale, graphic training simulator of a three-loop, 1000 MWe Pressurised-Water Reactor. The process simulated corresponded to the plant in which the trainees normally worked, but the humansystem interface was not identical.

\section{Participants}

In all, 21 licensed NPP operators from a Nordic plant participated in the study. The average age of the operators was 41 years, ranging from 29 to 60 years. 16 of the operators were reactor operators or assisting reactor operators, and five were shift managers or shift engineers. The participants had in average worked in nuclear power operation for 17 years, ranging from 7 to 40 years. All participants were male, and for this reason, the trainees will in the following be referred to as he or him. For the participants, the CIAU training session constituted an integrated part of a refresher training course, which they had volunteered to take part in.

\section{Scenario structure}

The structure of a CIAU training session is shown in Figure 2. In the present evaluation study, a training session lasted around one hour. A session contained two overall parts: scenario part 1 and scenario part 2 . In scenario part 1 , the trainee received no coaching. In scenario part 2 , the trainee received coaching. As the human-system interface in the simulator was not identical to the one the trainees normally used, assistance was offered with action implementation.

A training session began with a shift hand-over session, where the trainee obtained information about the state of the plant and received orders from the Operation Manager. Following this, the simulator was started, and the trainee engaged in developing a strategy for how to handle the situation at hand, while thinking aloud. The two coaches observed the trainee's performance. When the trainee started to implement the strategy he had developed, the simulator was frozen, and a coach asked the trainees to answer five generic questions marking the end of scenario part 1 . In the break following scenario part 1, both coaches and trainees filled-in the first part of their respective evaluation form. Then, the coaches completed the operator profile and determined how to frame the coaching process, and scenario part 2 began. The trainee was informed that he would have the opportunity to adjust the strategy he developed during scenario part 1 after completing the scenario. In scenario part 2 , the trainee received coaching. The simulator was running (e.g. to assess the impact of different actions) or frozen, depending on the need. When the scenario was completed, the trainee informed if he wanted to adjust the strategy he developed during scenario part one, and then the second part of the evaluation forms were filled in. After completion of the training session, a debriefing session was performed. During the study, debriefing was carried out as a group session at the end of a training day.

\section{Scenarios}

Each trainee performed one scenario. In all, two different scenarios were applied. Both scenarios lasted for a little under one hour and implied that the trainees would engage in an on-going emergency, which had potentials for escalation.

Scenario 1 involved a severe disturbance in the main coolingwater intake to the turbine condensers. It caused a high pressure in the condensers and in turn resulted in turbine and reactor trip and blocked the possibilities for steam dump. All safety valves opened to release residual power from the reactor, and all the safety valves closed again as they should, except for one valve. This valve was stuck in open position, which leads to an uncontrolled cool-down of the reactor coolant system (i.e. the primary side). Multiple tube leaks in two of the steam generators occurred, in combination with a secondary leak in one of the steam generators, which resulted in an uncontrolled release of radiation into the atmosphere. At the end of the hand-over, the trainee was informed that the Operation Manager ordered him to urgently start to cool down the reactor coolant system with the maximum allowed cool-down rate.

Scenario 2 involved an unintentional trip of the reactor combined with the condenser steam-dump system being unavailable. These occurrences were both due to human errors of maintenance personnel. These errors implied that the steam generator safety valves and the reactor pressuriser's release valve opened and closed frequently causing pressure spikes in the reactor-coolant system and a break to a connected auxiliary system. At the end of the hand-over, the trainee was informed that the Operation Manager ordered him to reduce the radioactive steam releases outside containment as soon as possible. This scenario was considered by the coaches to be somewhat more complex than scenario 1 .

\section{Operating procedures}

The trainees had no operating procedures available, expect for one (scenario 1) or two (scenario 2) procedure steps. 
Citation: Skjerve AB, Holmgren L (2018) Training Licensed Nuclear Power Plant Operators for Handling Unforeseen Accident Events: Development and Assessment of a Training Approach Based on Adaptive Expertise Theory. Arts Social Sci J 9: 385. doi: 10.4172/2151-6200.1000385

Page 6 of 9

\section{Results and Discussion}

\section{Testing scenarios for novelty}

To ensure that training will come to promote the development of adaptive expertise, and accordingly that the CIAU training approach can be assessed for its potential usefulness, the scenarios should be perceived as novel by the trainees. To assess the perceived novelty of the two scenarios, data obtained from two items in the evaluation form of the trainees (Table 1).

The results showed that the scenarios were not experienced as entirely novel by the trainees. This was expected (above Section) still, for both scenarios, the trainees agreed more than disagreed with the statements that the scenarios contained elements that were completely novel and resulted in a completely novel situation. For scenario 1 , the average score was 4.8 out of 7 and for scenario 2,5 out of 7 . When analysing the trainees' responses to the generic question: "Have you experienced similar situations (as the one faced in the present scenario) in simulator training sessions before?" (above Section), the same pattern was found. For example, one trainee stated 2: "Yes, I have run a similar scenario with a leakage in the reactor coolant system. But then again, this scenario is different. It is the same, but there are much more issues to attend to now." Another trainee stated: "No, I don't recall this particular scenario, but we have probably run something like that in the simulator.... maybe". Overall, results were interpreted to imply that both scenarios were sufficiently novel to proceed with the assessments of the potential usefulness of CIAU training for promoting operators' ability to hand unforeseen events.

\section{The potential usefulness of CIAU training for promoting li- censed NPP operators' ability to handle unforeseen events}

The potential usefulness of CIAU training for promoting licensed NPP operators' ability to handle unforeseen events (research question 1) was addressed based on responses provided by the trainees and the coaches in their respective evaluation forms. Following scenario part 2 , both the trainees and the coaches rated the impact of coaching on the trainee's level of adaptive expertise using a response scale with 15 points. The first, ranging from -7 to -1 signified a negative impact. The last 1 to 7 signified a positive impact. A score of 0 signified 'no impact'. The respondents all assessed that CIAU training had a positive impact on the trainees' level of adaptive expertise (Table 2). The assessed degree of positive impact, however, varied between the trainees and the coaches: The trainees' assessed the positive impact to be higher than did the coaches, with average scores of 5 and 3.5, respectively.

A reason for the difference might be that the trainees obtained more insights than the coaches were able to observe within the relatively short time frame of each training session.

Another indication of the potential usefulness of CIAU training was to compare the coaches' assessment of the quality of the trainees' strategies as assessed prior to and following coaching. Both assessments were made using a 7-point response scale. To calculate the impact of coaching on the trainees' strategies, i.e. the coaching impact score, the scores provided following scenario part 2 were subtracted/added to the scores provided prior to scenario part 1 , and the number signifying the distance between the two were added to the score provided after scenario part 1 (Table 3 ).

A comparison of the coaches' ratings of the quality of the trainees' strategies prior to coaching and the impact of coaching on the quality of

\begin{tabular}{|c|c|c|c|c|c|c|c|c|}
\hline \multirow[t]{2}{*}{ Item } & \multicolumn{2}{|c|}{ Mean } & \multicolumn{2}{|c|}{ Min. } & \multicolumn{2}{|c|}{ Max. } & \multicolumn{2}{|c|}{ Std.Dev. } \\
\hline & Sc. 1 & Sc. 2 & Sc. 1 & Sc. 2 & Sc. 1 & Sc. 2 & Sc. 1 & Sc. 2 \\
\hline $\begin{array}{l}\text { The scenario contained one } \\
\text { or more elements/issues that } \\
\text { were completely novel to me }\end{array}$ & 4.4 & 5.3 & 2.0 & 4.0 & 6.0 & 7.0 & 1.4 & 0.9 \\
\hline $\begin{array}{l}\text { The combination of elements/ } \\
\text { issues in the scenario resulted } \\
\text { in a situation that was } \\
\text { completely novel to me }\end{array}$ & 5.2 & 4.7 & 3.0 & 1.0 & 7.0 & 7.0 & 1.4 & 1.9 \\
\hline Average score & 4.8 & 5 & 2.5 & 2.5 & 6.5 & 7 & 1.4 & 1.4 \\
\hline
\end{tabular}

Table 1: The scenarios degree of novelty as perceived by the trainees $(n=21)$ using a 7-point response scale, where "1" signifies completely disagree and "7" completely agree. Legend: Sc. 1: Scenario 1; Sc. 2: Scenario 2.

\begin{tabular}{|c|c|c|c|c|c|c|}
\hline Score type & Scenario & Valid N & Mean & Min & Max & Std. Dev \\
\hline Trainees & 1 & 9 & 5 & 1 & 7 & 1.8 \\
\hline Trainees & 2 & 12 & 5.1 & 3 & 7 & 1.2 \\
\hline Coaches & 1 & 9 & 3.6 & 2 & 4.5 & 0.7 \\
\hline Coaches & 2 & 12 & 3.4 & 2.5 & 5 & 0.9 \\
\hline
\end{tabular}

Table 2: CIAU training were assessed to a positive impact on operators conceptual understanding of the plant by both trainees and coaches. All scores provided were positive signifying a positive impact, ranging from " 1 " to " 7 ", where "7" signifies the most positive impact.

\begin{tabular}{|c|c|c|c|c|c|}
\hline Item & Valid N & Mean & Min & Max & Std. Dev \\
\hline Prior to coaching & 21 & 4.23 & 3 & 6 & 0.94 \\
\hline Following coaching & 21 & 5.4 & 4 & 7 & 0.86 \\
\hline Coaching impact score & 21 & 9.7 & 7 & 12.5 & 1.53 \\
\hline
\end{tabular}

Table 3: The coaches' assessments of the trainees' strategies prior to coaching and following coaching.

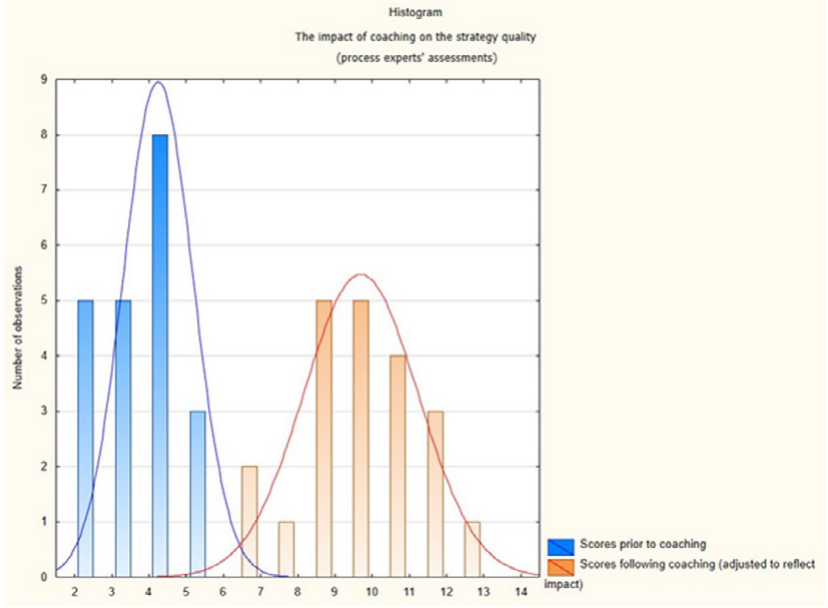

Figure 3: The impact of coaching on the quality of the strategies developed by the trainees.

the strategy is shown in Figure 3. The results indicate that coaching in all cases succeeded in improving some aspects of the trainee's strategy.

The level of adaptive expertise of each trainee was indicated based on the average score on three items in the coaches' evaluation form, which was provided after scenario part 1 . The coaches assessed the trainee's level of domain-specific skills, metacognitive skills and innovative skills. Each item was associated with a 7-point response scale. To obtain insights into whether the initial adaptive expertise level might impact the potential benefits of CIAU training, the trainees level of adaptive expertise was correlated with the extent to which the trainees were able to benefit from coaching, as assessed by the coaches in their 
Citation: Skjerve AB, Holmgren L (2018) Training Licensed Nuclear Power Plant Operators for Handling Unforeseen Accident Events: Development and Assessment of a Training Approach Based on Adaptive Expertise Theory. Arts Social Sci J 9: 385. doi: 10.4172/2151-6200.1000385

Page 7 of 9

evaluation form following scenario part 2: A positive correlation was found, $\mathrm{r}=0.65, \mathrm{p} \leq 0.005$, with a $\mathrm{R}^{2}=0.42$. The level of adaptive expertise score was then correlated with trainee age and experience from NPP operation, to explore if the level of adaptive expertise could simply reflect a higher experience level. However, no correlations were found. These results could indicate that trainees which as a starting point have a higher level of adaptive expertise might benefit most from CIAU training.

\section{Competencies improved by CIAU training}

To understand what competencies, the trainees improved during CIAU training (research question 2), the trainees and the coaches were asked to document what competencies the trainees had mainly promoted following scenario part 2, using a free text response format. The assessments of the trainees and coaches, which over all corresponded, are summarized in Table 4.

As can be seen from Table 4, the competencies improved included conceptual domain-specific skills (e.g., thermo-and flow dynamics) and metacognitive skills (e.g. strategy thinking balancing the pros and cons). Improvement of innovative skills may further to some extent be reflected in learning materials concerning increased ability consider the state of the plant " $\ldots$ in a longer and deeper perspective". As this training material covers competencies that are traditionally considered to be elements of adaptive expertise, the results indicate that CIAU training succeeded in promoting adaptive expertise.

\section{Trainees' assessments of the CIAU training approach}

The trainees' assessments of the CIAU training approach (research question 3) was explored based on their responses to two items in the evaluation form filled in following scenario part 2: One item covered what aspects they appreciated most and one what aspects they found most demanding about the training approach. In both cases, the response format was free text. Typically, each trainee provided one or two viewpoints on both items. The trainees also provided feedback on this topic during the debriefing session. A summary of the trainees' assessments can be found in Table 5 .

The aspect of CIAU training the trainees appreciated most was the possibility, the possibility it provided for them to work out a solution/ strategy to an operational issue on their own. This was reported as an advantage by more than half of the trainees (i.e. 14 trainees) and stated several times during the debriefing sessions. The trainees found that working out a solution on their own - guided by the coaches when needed - provided them with a deeper insight into the working of the plant and allowed them to understand all part of the reasoning processes leading up to the particular solution in details, including why some alternatives would not work.

\section{Trainees' Assessments}

To see a situation in the plant in a longer-term and deeper perspective.

To accurately assess situations and Strategy thinking, balancing the pros and maintain an overview.

Tucked away knowledge comes to the surface.

Thermo-and flow dynamics in the plant.

Improved insights into the plant's capacity and limitations.

Table 4: The trainees' and coaches' assessments of competencies the trainees improved from participating in CIAU training.

\section{Most appreciated aspects}

To have the opportunity to work out solutions/strategies on your own.

Coaching helps you to increase

your insights into the situation and ensures that you are guided back on the right-track, if needed.

Coaching results in "Eureka"experiences and opens the door to alternative ways of thinking.

The opportunity to think outside the box.

The possibility to think about something in-depth, makes you understand the necessity of upholding a questioning attitude.

Table 5: A summary of the trainees' assessment of the CIAU training approach.

The aspect of CIAU training the trainees found to be the most demanding was that they had little time available for developing a strategy for bringing the plant back to a safe state. This aspect was reported in the evaluation form by more than half of the trainees (i.e. 12 trainees). In future settings, then, it might be advisable to expand a CIAU training sessions somewhat to allow trainees' more time for strategy development. Still, CIAU training sessions are highly demanding from a cognitive perspective, requiring focused concentration throughout the training session, and for this reason it is likely that training effectiveness might be reduced if a training session exceeds a certain time limit.

The trainees' evaluation form contained the following statement posed following scenario part 2: "I would like to take part in more of this type of training". The statement was associated with at 7-point response, ranging from 1 (completely disagree) to 7 (completely agree). The trainees' average score of on the item was 6.5, ranging between 5 and 7 . Thus, overall the trainees appreciated taking part in CIAU training

\section{Conclusion and Future Research}

The study suggested that CIAU training showed a potential for promoting licensed NPP operators' ability to handle unforeseen events by advancing their level of adaptive expertise (research question 1). The support for this was that both trainees and coaches judged that CIAU training had a positive impact on the trainees' conceptual understanding of the plant processes, and that the strategies developed by the trainees following coaching were improved, as compared the strategies they developed prior to coaching. These findings, thus, also demonstrated the applicability of adaptive expertise theory in the domain of NPP operation. CIAU training improved both domainspecific skills and metacognitive skills, and thus the basis for innovative solutions (research question 2). The aspect of CIAU training that the trainees appreciated was the possibility for working out solutions/ strategies on their own. The aspect they found to be most demanding was the limited time available for developing a strategy. The trainees were positive to engaging in more CIAU training.

The study assessed the potential usefulness of CIAU training for promoting licensed NPP operators' ability to handle unforeseen events. It also had a set of limitations. The study was based on data obtained in a Nordic country, and the culture in Nordic countries are characterised by a low power distance [46]. It could be that it is easier to establish a learner-friendly environment of the type required by CIAU training in cultures that are characterised by a lower rather than a higher power 
Citation: Skjerve AB, Holmgren L (2018) Training Licensed Nuclear Power Plant Operators for Handling Unforeseen Accident Events: Development and Assessment of a Training Approach Based on Adaptive Expertise Theory. Arts Social Sci J 9: 385. doi: 10.4172/2151-6200.1000385

Page 8 of 9

distance. Also, the trainees' entry level of adaptive expertise - at least as assessed in the study - might have impacted the outcome of the study. A positive correlation was found between entry level of adaptive expertise and ability to benefit from CIAU coaching, and possibly, and there it is not clear if the entry level of adaptive expertise in the overall population of licensed NPP operators, differ from that of the trainees who took part in the study.

To obtain more insights into the usefulness of CIAU training, more data on training effectiveness is needed. In addition, to compare licensed NPP operators' ability to handle scenarios containing unforeseen events unassisted by the coaches prior to and post several CIAU training sessions, it is also necessary to assess training transfer, i.e., the extent to which the trainees will apply the lessons learned during training in their normal work environment. In particular, it will be useful to address the extent to which the trainees apply the mental strategies encouraged during CIAU training in their normal work environment. Continuous application of these strategies may contribute to cultivate and uphold a questioning attitude, which has been described as one of the key important safety principles in a strong safety culture [47]. In addition, it may promote operators in continuously improving their level of competence. For this reason, transfer of CIAU training might potentially come to promote a way of working in the every-day work environment of the operators that will further contribute to promote their ability to handle unforeseen events.

\section{Acknowledgements}

We sincerely thank all operators who participated in the study and willingly shared their experiences and assessments with us, as well as the deputy operation managers, who acted as subject during the pilot study. We would like to thank the training organisation, who allowed us to use their training simulator. We will also, thank Bjarne Widheden (KSU AB) and Christer Nihlwing (IFE), and in particular Olof Berntsson (KSU AB) for invaluable practical support and for sharing his ideas and reflections with us during the study.

\section{References}

1. Gibson JJ (1964) The contribution of experimental psychology to the formulation of the problem of safety - a brief for basic research. In: Haddon W, Suchman EA, Klein D (eds.) Accident research: methods and approaches. Harper \& Row New York, NY.

2. Haddon W (1980) The basic strategies for reducing damage from hazards of all kinds. Hazard Prevention 16: 8-12.

3. Turner BA (1978) Man-made Disasters. Wykeham Science Press, London.

4. Turner BA (1994) Causes of disaster: sloppy management. Br J Management 5: 215-219.

5. Turner BA, Pidgeon NF (1997) Man-made Disasters. (2ndedn), ButterworthHeinemann, London.

6. Perrow C (1984) Normal Accidents: Living with High Risk Technologies. Princeton University Press, Princeton.

7. La Porte TR, Consolini P (1991) Working in practice but not in theory: theoretical challenges of high-reliability organizations. J Public Adm Res Theory 1: 19-47.

8. Rasmussen J (1997) Risk management in a dynamic society: a modelling problem. Safety Science 27: 183-213.

9. Hollnagel E, Woods DD, Leveson NC (2006) Resilience engineering: Concepts and precepts. Ashgate, Aldershot, UK.

10. World Nuclear Association (2018) Nuclear Power in the World Today.

11. Mahaffey J (2014) Atomic Accidents: A History of Nuclear Meltdowns and Disasters: From the Ozark Mountains to Fukushima. Pegasus Books, New York

12. IAEA (1996) Defense in depth in nuclear safety (INSAG-10). A report by the international nuclear safety advisory group. International Atomic Energy Agency, Vienna
13. Kemeny JG, Babbitt B, Haggerty PE, Lewis C, Marks P et al. (1979) The President's Commission on the Accident at Three Mile Island. The need for change: Legacy of TMI.

14. IAEA (1991) Use of plant specific PSA to evaluate incidents at nuclear power plants (TECDOC-611). International Atomic Energy Agency, Vienna.

15. Haage M (2014) The Human and Organizational Part of Nuclear Safety Presentation at the Nuclear Safety Culture Workshop. A Systems Approach to Safety Culture.

16. Bainbridge L (1983) Ironies of automation. Automatica 19: 755-779.

17. Kaarstad M, Torgersen GE (2017) Is it Possible to Assess an Organization's Preparedness for the Unforeseen? Development and Evaluation of a Methodology. Arts Social Sci J 8: 254.

18. Kurokawa K, Ishibashi K, Oshima K, Sakiyama H, Sakurai M, et al. (2012) The National Diet of Japan. The official report of the Fukushima Nuclear Accident Independent. Investigation Commission. The National Diet of Japan, Tokyo.

19. Hatano G, Inagaki K (1986) Two courses of expertise. In: Stevenson H, Azuma $\mathrm{H}$, Hakuta K (eds.) Child development and education in Japan. Freeman, San Francisco, pp: 262-272.

20. Kahneman D (2011) Thinking, fast and slow. Farrar, Straus and Giroux, New York.

21. Rasmussen J (1983) Skills, rules, knowledge; signals, signs, and symbols and other distinctions in human performance models. IEEE Transactions on Systems, Man and Cybernetics 13: 257-266.

22. Bada SO (2015) Constructivism Learning Theory: A Paradigm for Teaching and Learning. IOSR Journal of Research \& Method in Education 5: 66-70.

23. Institute for Energy Technology (2008) 50 Years of Safety-related ResearchThe Halden Project 1958-2008.

24. Skjerve AB, Holmgren L, Widheden B (2015) Towards an Approach for Training Nuclear Power Plant Control-Room Crews in Handling Unforeseen Events. In: Podofillini L, Sudret B, Stojadinović B, Zio E, Kröger W (eds.) Safety and Reliability of Complex Engineered Systems. Taylor \& Francis Group, London, pp: 3895-3902.

25. Hatano G, Oura Y (2003) Commentary: Reconceptualizing School Learning Using Insight from Expertise Research. Educational Research 32: 26-29.

26. Bell BS, Kozlowski SWJ (2008) Active learning: Effects of core training design elements on self-regulatory processes, learning and adaptability. The Journal of Applied Psychology 93: 296-316

27. Carbonell KB, Stalmeijer RE, Könings KD, Segers $M$, van Merriënboer JJG (2014) How experts deal with novel situations: A review of adaptive expertise. Educational Research Review 12: 14-29.

28. Bransford JD, Brown AL, Cocking RR (2000) How People Learn: Brain, Mind Experience and School. National Academy Press, Washington D.C.

29. Smith EM, Ford K, Kozlowski SWJ (1997) Building Adaptive Expertise: Implications for Training Design Strategies. In: Quiñones MA, Ehrenstein A (eds.) Training for a rapidly changing workplace: applications of psychological research. American Psychological Association, Washington, DC, pp: 89-118.

30. Carbonell KB, Könings KD, Seger M, van Merriënboer JJG (2015) Measuring adaptive expertise: development and validation of an instrument. European Journal of Work and Organizational Psychology 252: 167-180.

31. Paul R, Elder L (2006) The Miniature Guide to Critical Thinking, Concepts and Tools. The Foundation for Critical Thinking

32. Ausubel DP (1960) The use of advance organizers in the learning and retention of meaningful verbal material. Journal of Educational Psychology 51: 267-272.

33. Flavell JH (1979) Metacognition and cognitive monitoring: A new area of cognitive-developmental inquiry. American Psychologist 34: 906-911.

34. Bonwell C, Eison J (1991) Active Learning: Creating Excitement in the Classroom AEHE-ERIC Higher Education Report.

35. Bruner JS (1961) The act of discovery. Harvard Educational Review 31: 21-32.

36. Clark RE, Voogel A (1985) Transfer of training principles for instructional design. Education Communication and Technology Journal 33: 113-123.

37. Clark RC (2015) Evidence-based training methods. A guide for training professionals (2ndedn), ASTD Press, Alexandria, VA 
Citation: Skjerve AB, Holmgren L (2018) Training Licensed Nuclear Power Plant Operators for Handling Unforeseen Accident Events: Development and Assessment of a Training Approach Based on Adaptive Expertise Theory. Arts Social Sci J 9: 385. doi: 10.4172/2151-6200.1000385

38. Vygotsky LS (1978) Mind in society: The development of higher psychological processes. Harvard University Press, Cambridge, MA.

39. Rogers A, Horrocks N (2010) Teaching Adults. (4thedn), Open University Press, Maidenhead.

40. Weisaeth L (1985) Traumatiske kriser og katastrofer. In: Retterstoel N, Weisaeth L (eds.) Katastrofer og kriser. Universitetsforlaget Oslo, pp: 55-75.

41. Kirkpatrick DL (1998) Evaluating Training Programmes: The Four Levels. (2ndedn), Berrett-Koehler Publishers, San Francisco, California.

42. Bates R (2004) A critical analysis of evaluation practice: the Kirkpatrick mode and the principles of beneficence. Evaluation and Program Planning 27: 341 347.

43. Kowalski TKM, Vaught C, Mallett LG, Brnich MJ, Reinke DC, et al. (2004) Safety and Health Training for an Evolving Workforce: An Overview from the Mining Industry. Information Circular/2004, IC 9474. DHHS (NIOSH) Publication No. 2004-155. National Institute for Occupational Safety and Health, Cincinnati, $\mathrm{OH}$.

44. IAEA (1996) Nuclear Power Plant Personnel Training and its Evaluation Technical Reports Series no. 380, IAEA, International Atomic Energy Agency, Vienna.

45. NUREG-1122 (1998) Knowledge and Abilities Catalog for Nuclear Power Plant Operators: Pressurized Water Reactors. Revision 2. Initial Report. US Nuclear Regulatory Commission, Maryland.

46. Hofstede G (2011) Dimensionalizing Cultures: The Hofstede Model in Context Online Readings in Psychology and Culture Vol. 2.

47. Guide S (2006) Application of the Management System for Facilities and Activities. IAEA Safety Standards Series No. GS-G-3.1, International Atomic Energy Agency, Vienna. 